UDC: 517.938

\title{
ON NUMERICAL SOLUTIONS OF LINEAR FRACTIONAL DIFFERENTIAL EQUATIONS
}

\author{
YLLDRITA SALIHI ${ }^{1}$, GJORGJI MARKOSKI ${ }^{2}$, AND ALEKSANDAR GJURCHINOVSKI $^{3}$
}

\begin{abstract}
Fractional differential equations have excited considerable interest recently, both in pure and applied mathematics. In this paper, we apply Fractional Adams-Bashforth Method (FAB), Fractional Adams-BashforthMoulton Method (FABM) and Fractional Multistep Differential Transform Method (FMDTM), for obtaining the numerical solutions of two distinct linear systems of fractional differential equations with fractional derivatives described in the Caputo sense. The numerical results for the three methods are compared with the exact solution for each linear system by using the relative difference between the exact and the approximate solution at each integration point. The results are given both graphically and tabularly, concluding that, aside from occasional non-monotoncity for small time values, all three numerical methods gradually diverge from the exact solution with increasing integration time, and the superiority of each numerical method over the others depends on the particular system under investigation.
\end{abstract}

\section{INTRODUCTION}

Fractional differential equations have become especially interesting in the past decade due to their numerous applications in describing variety of phenomena in many areas of physics and engineering [1, 2]. It has been shown that the spatialtemporal dynamics of variety of physical phenomena can be expressed reliably via equations containing derivatives and integrals of non-integer order. The fractional derivative is not uniquely defined, and in this paper we use the Caputo definition of fractional derivative [1, 2].

Most systems described by fractional differential equations do not have exact solutions, and finding analytical solutions of fractional differential equations could

2000 Mathematics Subject Classification. 34C28, 34A08, 74H15.

Key words and phrases. Fractional differential equations, Fractional Adams-Bashforth Method, Fractional Multistep Differential Transform Method, Fractional Adams-BashforthMoulton Method. 
be a formidable task. In majority of cases, it is only possible to provide a numerical approximation of the solution [2, 3].

In this paper, we use three different numerical methods to find approximate solutions of two distinct linear differential systems of fractional type. To solve the linear differential equations, we use Fractional Adams-Bashforth Method (FAB), Fractional Adams-Bashforth-Moulton Method (FABM) and Fractional Multistep Differential Transform Method (FMDTM) [4, 5, 6, 7, 8, The organization of this paper is as follows. The definitions and mathematical preliminaries of fractional calculus theory that we exploit in this paper are provided in Section 2. In Section 3 we give an overview of the used numerical methods and the details of the corresponding algorithms. In Section 4, we present two examples of linear fractional-order systems that are solved analytically in terms of one-parameter Mittag-Leffler functions which are generalizations of an exponential function. The obtained numerical results are then compared with the exact solutions for each example by using the relative difference between the exact and the approximate solution. The time-series are displayed both graphically and tabularly. The conclusions are given in Section 5. All the numerical examples are done by using the Wolfram Mathematica package. The notebook programs are available upon request.

\section{BASIC DEFINITIONS}

Definition 2.1. A real function $f(t), t>0$, is said to be in space $C_{\mu}, \mu \in R$ if thre exists a real number $p(>\mu)$, such that $f(t)=t^{p} f_{1}(t)$, where $f_{1}(t) \in C[0, \infty)$, and it is said to be in the space $C_{\mu}^{m}$ iff $f^{(m)} \in C_{\mu}, m \in N$.

Definition 2.2. The Riemman-Liouville fractional integral operator of order $\alpha \geq 0$ of a function $f(t) \in C_{\mu}, \mu \geq-1$ is defined as:

$$
J^{\alpha} f(t)=\frac{1}{\Gamma \alpha} \int_{0}^{t}(t-\tau)^{\alpha-1} f(\tau) d \tau
$$

Definition 2.3. The Caputo fractional derivative of a function $f(t)$ is defined by:

$$
D_{t}^{\alpha} f(t)=J^{n-\alpha} D_{t}^{n} f(t)=\frac{1}{\Gamma(n-\alpha)} \int_{0}^{t}(t-\tau)^{n-\alpha-1} f^{(n)}(\tau) d \tau
$$

for $n-\alpha<\alpha \leq n, n \in N, t>0$, and $f(t) \in C_{-1}^{m}$.

Remark 1. In the definition for Caputo fractional derivative we first differentiate $f(t) n$-times, then integrate it $n-\alpha$ times. If $f(t)$ is $n$-times differentiable, then the $\alpha$-th order derivative will exist, where $n-\alpha<\alpha \leq n$, otherwise this definition is not applicable. Two main advantages of this definition are: i). fractional derivative of a constant is zero; ii). fractional differential equation of Caputo type has initial conditions of classical non-integer derivative type, in contrast to fractional differential equation of Riemann-Liouville type, where initial conditions are of fractional type. 
Two basic properties of Caputo fractional derivative that immediately follow from Definition 2.3 are:

$$
\begin{aligned}
& J^{\alpha} D_{t}^{\alpha} f(t)=f(t)-\sum_{k=0}^{n-1} f^{(k)}\left(0^{+}\right) \frac{x^{k}}{k !}, \quad t>0, \\
& D_{t}^{\alpha} J^{\alpha} f(t)=f(t) .
\end{aligned}
$$

Definition 2.4. The two parameter Mittag-Leffler function is defined (in series form) as:

$$
E_{\alpha, \beta}(z)=\sum_{k=0}^{\infty} \frac{z^{k}}{\Gamma(\alpha k+\beta)}, \quad z \in C, \quad \operatorname{Re}(\alpha), \beta>0
$$

If $\beta=1$ then we have a one-parameter Mittag-Leffler function

$$
E_{\alpha}(z)=\sum_{k=0}^{\infty} \frac{z^{k}}{\Gamma(\alpha k+1)}
$$

The Mittag-Leffler functions are generalizations of the exponential function, and solutions of fractional order linear differential equations are often expressed in terms of Mittag-Leffler functions.

Definition 2.5. The $m$-th derivative of Mittag-Leffler function, for $m=$ $0,1,2, \ldots$, are given by:

$$
E_{\alpha, \beta}^{(m)}(z)=\sum_{k=0}^{\infty} \frac{(k+m) ! z^{k}}{k ! \Gamma(\alpha k+\alpha m+\beta)}
$$

\section{Numerical Methods}

For the initial value problem

$$
D_{t}^{\alpha} y(t)=f(t, y(t)), \quad y^{(k)}(0)=y_{0}^{(k)}
$$

$(k=0,1,2, \ldots,\lceil\alpha\rceil-1)$, we will construct three numerical methods, FAB, FABM and FMDTM, assuming that a solution of (3.1) is sought on some time interval $[0, T]$.

3.1. Fractional Adams-Bashforth Method. In order to assure the existence and uniqueness of the solution to 3.1 , it is assumed that $f(t, y(t))$ is continuous and fulfils the Lipschitz condition with respect to the second variable. On $[0, T]$, for a uniform grid $t_{j}=h j(j=0,1, \ldots, N)$ and a constant time step denoted by $h=\frac{T}{N}$, the goal is to approximate solution values $y_{j} \approx y\left(t_{j}\right)$ at the grid points. According to the theorem of existence and uniqueness of the solution, initial value problem (3.1) can be reformulated in terms of the weakly-singular Volterra integral equation:

$$
y(t)=\sum_{k=0}^{\lceil\alpha\rceil-1} \frac{y_{0}^{(k)}}{k !} t^{k}+\frac{1}{\Gamma(\alpha)} \int_{0}^{t}(t-\tau)^{\alpha-1} f(\tau, y(\tau)) d \tau .
$$


The method immediately suggests a numerical approach in solving 3.2 . The main part of the algorithm for FAB is the iteration formula

$$
y[j]=\sum_{k=0}^{\lceil\alpha\rceil-1} \frac{(j h)^{k}}{k !} y_{0}^{(k)}+\frac{h^{\alpha}}{\Gamma(\alpha+1)} \sum_{k=0}^{j-1} b[j-k] f(k h, y[k])
$$

where $b[j-k]$ are the weights which depend only on the difference $(j-k)$ because of the convolution structure of $b_{k, j}$ :

$$
b_{k, j}=\frac{(j-k)^{\alpha}-(j-k-1)^{\alpha}}{\Gamma(\alpha+1)} .
$$

3.2. Fractional Adams-Bashforth-Moulton Method. The discussed FAB method is a natural candidate for a predictor in the process of constructing the predictorcorrector method FABM (the Adams-Moulton method can be constructed in similar way like FAB).

The FABM method is said to be Predict-Evaluate-Correct-Evaluate type because an initial approximation $p$, the so-called predictor, is evaluated first:

$$
p=\sum_{k=0}^{\lceil\alpha\rceil-1} \frac{(j h)^{k}}{k !} x_{0}^{(k)}+\frac{h^{\alpha}}{\Gamma(\alpha+1)} \sum_{k=0}^{j-1} b[j-k] f(k h, y[k]) .
$$

Then the method gives the corrector formula:

$$
\begin{aligned}
y[j]= & \sum_{k=0}^{\lceil\alpha\rceil-1} \frac{(j h)^{k}}{k !} y_{0}^{(k)} \\
& +\frac{h^{\alpha}}{\Gamma(\alpha+2)}\left(f(j h, p)+\left((j-1)^{\alpha+1}-(j-\alpha-1) j^{\alpha}\right) \cdot f(0, y[0])+\right. \\
& \left.+\sum_{k=0}^{j-1} a[j-k] f(k h, y[k])\right)
\end{aligned}
$$

where $p$ represents FAB, which in this case acts like a predictor. The weight $a[j-k]$ in the corrector $y[j]$ is given by

$$
a[j-k]= \begin{cases}\frac{(j-1)^{\alpha+1}-(j-\alpha-1) j^{\alpha}}{\Gamma(\alpha+2)}, \quad \text { if } j=0 \\ \frac{(j-k+1)^{\alpha+1}+(j-k-1)^{\alpha+1}-2(j-k)^{\alpha+1}}{\Gamma(\alpha+2)}, & \text { if } j \in[1, k-1] \\ 1, \quad \text { if } j=k & \end{cases}
$$

3.3. Fractional Multistep Differential Transform Method. The basic definition and the fundamental theorems of the differential transform method (DTM) and its applicability for various kinds of differential equations are given in the following paragraphs.

The differential transform of the $k$-th derivative of function $f(t)$ is defined as:

$$
F(k)=\frac{1}{k !}\left[\frac{d^{k} f(t)}{d t^{k}}\right]_{t=t_{0}}
$$


where $f(t)$ is the original function and $F(k)$ is the transformed function. The differential inverse transform of $F(k)$ is defined by

$$
f(t)=\sum_{k=0}^{\infty} F(k)\left(t-t_{0}\right)^{k}
$$

From 3.8 and 3.9 , we get $f(t)=\sum_{k=0}^{\infty} \frac{\left(t-t_{0}\right)^{k}}{k !} \frac{d^{k} f(t)}{d t^{k}}$, which implies that the concept of differential transform is derived from Taylor series expansion.

To describe the FMDTM, we consider the initial value problem (3.1). In actual applications of DTM, approximate solution of the initial value problem (3.1) can be expressed by the finite series in the form

$$
y(t)=\sum_{n=0}^{N} a_{n} t^{n}, \quad t \in[0, T]
$$

Assume that the interval $[0, T]$ is divided into $M$ subintervals $\left[t_{m-1}, t_{m}\right], m=$ $1,2, \ldots, M$ of equal step size $h=\frac{T}{M}$ by using the nodes $t_{m}=m h$. The main idea of the FMDTM is in the following. First, if we apply DTM to (3.1) over the interval $\left[0, t_{1}\right]$, we will obtain the following approximate solution:

$$
y_{1}(t)=\sum_{n=0}^{K} a_{1 n} t^{n}, \quad t \in\left[0, t_{1}\right]
$$

using the initial conditions $y_{1}^{(k)}(0)=c_{k}$.

For $m \geq 2$, at each subinterval $\left[t_{m-1}, t_{m}\right]$ we will use the initial conditions $y_{m}^{(k)}\left(t_{m-1}\right)=$ $y_{m-1}^{(k)}\left(t_{m-1}\right)$ and apply DTM to 3.1$)$ over the subinterval, where $t_{0}$ in 3.8 is replaced by $t_{m-1}$. The process is repeated and generates a sequence of approximate solutions $y_{m}(t), m=1,2, \ldots, M$, for the solution $y(t)$ :

$$
y_{m}(t)=\sum_{n=0}^{K} a_{m n}\left(t-t_{m-1}\right)^{n}, \quad t \in\left[t_{m}, t_{m+1}\right]
$$

where $N=K M$. In fact, FMDTM assumes the following solution:

$$
y(t)= \begin{cases}y_{1}(t), & t \in\left[0, t_{1}\right] \\ y_{2}(t), & t \in\left[t_{1}, t_{2}\right] \\ \vdots & \\ y_{M}(t), & t \in\left[t_{M-1}, t_{M}\right]\end{cases}
$$

The new algorithm, FMDTM, is proven simple for computational performance for all values of $h$.

\section{Applications}

Example 4.1. As our first example, we consider the homogeneous linear fractional-order differential equation [9]

$$
D_{t}^{\alpha} x(t)=-x(t), x(0)=1, t>0
$$


for $0<\alpha \leq 1$, with its corresponding exact solution

$$
x(t)=-E_{\alpha}\left(t^{\alpha}\right),
$$

where $E_{\alpha}(\cdot)$ is the one-parameter Mittag-Leffler function of order $\alpha$. For the value of the fractional order we take $\alpha=0.98$ as a particular example, but the conclusions of our numerical analysis holds also for other values of $\alpha$ close to, but less than, 1 .
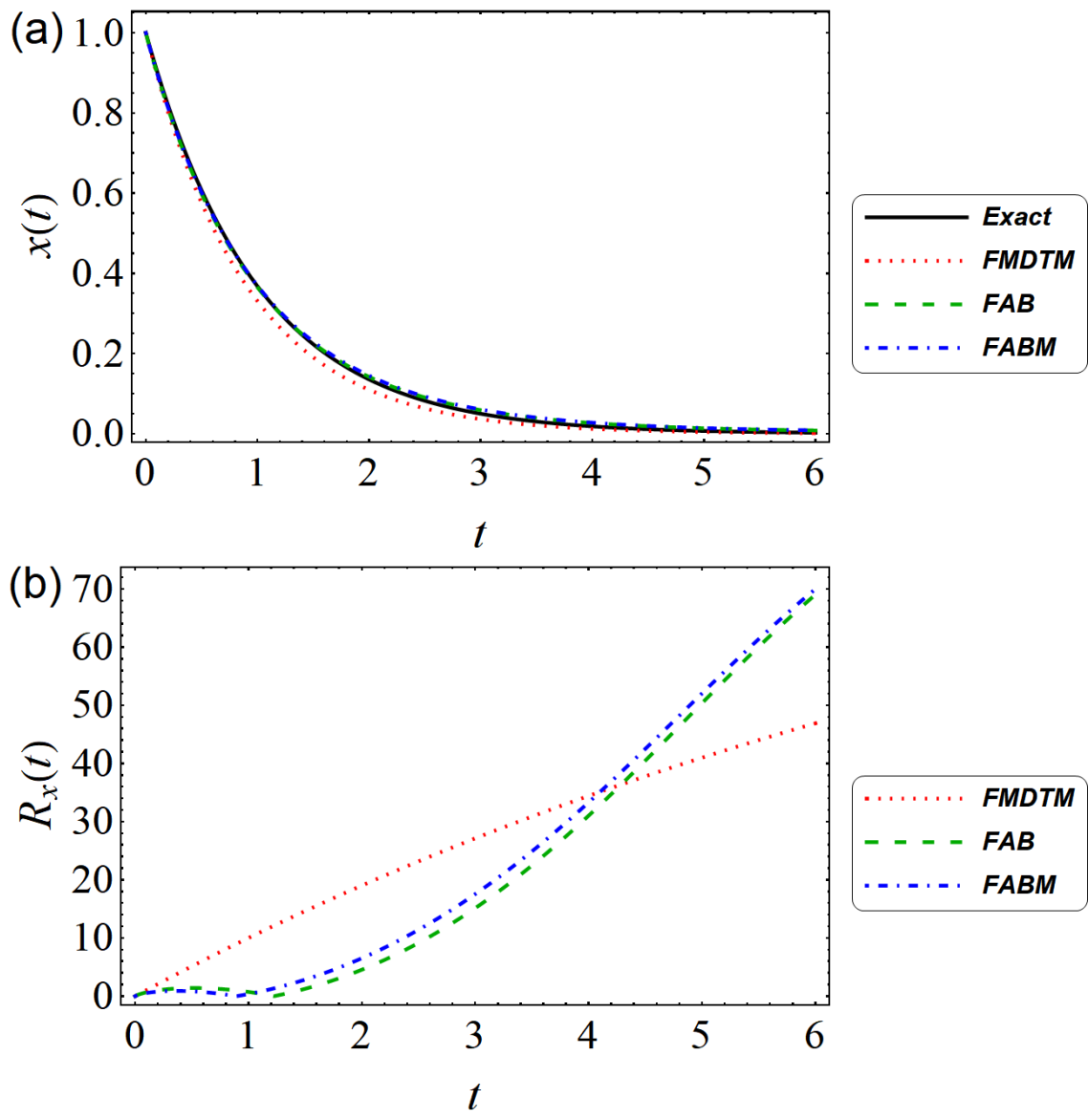

Figure 1. (a). Time-series $x(t)$ versus $t$ of the exact integration curve of Eq. 4.1 (black/solid line) and the approximation curves for FMDTM (dotted/red line), FAB (dashed/green line), and FABM (dot-dashed/blue line); (b). Relative difference $R_{x}(t)$ corresponding to FMDTM (dotted/red line), FAB (dashed/green line), and FABM (dotdashed/blue line). The fractional-order parameter is $\alpha=0.98$. In all simulations, initial condition $x(0)=1$, integration step size $h=0.01$ and $t \in[0,6]$. 
In Fig. 1a we depict the plots of $x(t)$ versus the time $t$ of the initial value problem (4.1) consisting of the exact integration curve (4.2) as well as the approximation time-series obtained from the three different numerical methods. The exact curve is depicted with a black (solid) line, and the dotted (red) line, the dashed (green) line and the dash-dotted (blue) line are the approximation time-series obtained with FMDTM, FAB, FABM respectively. In all numerical simulations, we take the integration step-size $h=0.01$, and the integration time-span is $t \in[0,6]$. It is seen that all the curves are characterized with a typical exponential-like decrease as $t \rightarrow \infty$. The deviation of the approximation curves from the exact one can hardly be perceived from the figure. To characterise this deviation, and hence the quality of particular approximation method, we introduce the relative difference parameter $R(t)$ defined as the absolute difference between the exact value $x_{\text {exact }}$ of the solution curve $x(t)$ at the fixed time $t$ and the approximate value $x_{\text {approx }}$ at the same instant of time divided by the maximum absolute value of these two numbers (expressed in percents \% by multiplying with 100):

$$
R_{x}(t)=\frac{\left|x_{\text {exact }}(t)-x_{\text {approx }}(t)\right|}{\max \left\{\left|x_{\text {exact }}(t)\right|,\left|x_{\text {approx }}(t)\right|\right\}} \cdot 100 \% .
$$

In Fig. $1 \mathrm{~b}$ we show the calculated plots of the relative difference $R_{x}(t)$ corresponding to FMDTM (dotted/red line), FAB (dashed/green line), and FABM (dot-dashed/blue line). Apart from a slight non-monotonic behavior at a small time interval at the begining, it is seen that all the curves $R_{x}(t)$ are monotonically increasing as the integration time becomes larger. This observation suggests that all three numerical methods gradually diverge from the exact solution with increasing integration time, and this divergence quickly becomes significant. Although all three methods approximate the exact solution in a practical range of relative deviations, in this case FMTDM is better approximation than FAB and FABM at large time points. These conclusions can also be extracted by looking at the tabluar values of the exact time-series $x_{\text {exact }}$ and the apprroximation time series $x_{F M D T M}, x_{F A B}$ and $x_{F A B M}$, along with the corresponding relative difference parameters $R_{F M D T M}, R_{F A B}$ and $R_{F A B M}$. The results are given in Table 1, where for clarity we show only the time-series at the beginning and at the end of the integration time-span.

Example 4.2. Our second example is the two-dimensional linear fractional differential system [10]

$$
\left\{\begin{array}{l}
D_{t}^{\alpha} x(t)=2 x(t)-y(t) \\
D_{t}^{\alpha} y(t)=4 x(t)-3 y(t)
\end{array},\right.
$$

where $0<\alpha \leq 1$. The system can be re-written in the matrix form

$$
\left(\begin{array}{l}
D^{\alpha} x(t) \\
D^{\alpha} y(t)
\end{array}\right)=\left(\begin{array}{ll}
2 & -1 \\
4 & -3
\end{array}\right)\left(\begin{array}{l}
x(t) \\
y(t)
\end{array}\right) \text {. }
$$

The eigenvalues of the matrix $\left(\begin{array}{ll}2 & -1 \\ 4 & -3\end{array}\right)$ are $\lambda_{1}=1$ and $\lambda_{2}=-2$, and their corresponding eigenvectors are $v_{1}=\left(\begin{array}{l}1 \\ 1\end{array}\right)$ and $v_{2}=\left(\begin{array}{l}1 \\ 4\end{array}\right)$ respectively. Therefore, 
TABLE 1. Partial data values of the time-series $x(t)$ for increasing $t$ of the exact solution of Eq. (4.1) and the numerical approximation values for FMDTM, FAB, and FABM, as well as the corresponding values for the relative difference $R_{x}(t)$ for each numerical method.

\begin{tabular}{c|c|c|c|c|c|c|c}
$t$ & $x_{\text {exact }}$ & $x_{F M D T M}$ & $x_{F A B}$ & $x_{F A B M}$ & $R_{F M D T M}$ & $R_{F A B}$ & $R_{F A B M}$ \\
\hline 0 & 1 & 1 & 1 & 1 & 0 & 0 & 0 \\
0.01 & 0.99005 & 0.989006 & 0.988882 & 0.989005 & 0.105467 & 0.117977 & 0.105505 \\
0.02 & 0.980199 & 0.978132 & 0.978191 & 0.978432 & 0.210822 & 0.204805 & 0.180233 \\
0.03 & 0.970446 & 0.967378 & 0.967728 & 0.968082 & 0.316067 & 0.280027 & 0.243503 \\
0.04 & 0.960789 & 0.956743 & 0.957449 & 0.957914 & 0.4212 & 0.347697 & 0.29932 \\
0.05 & 0.951229 & 0.946224 & 0.947332 & 0.947904 & 0.526223 & 0.409726 & 0.349573 \\
0.06 & 0.941765 & 0.935821 & 0.937364 & 0.938041 & 0.631135 & 0.467235 & 0.395369 \\
0.07 & 0.932394 & 0.925532 & 0.927536 & 0.928315 & 0.735936 & 0.520961 & 0.437437 \\
0.08 & 0.923116 & 0.915356 & 0.917841 & 0.91872 & 0.840626 & 0.571428 & 0.476291 \\
0.09 & 0.913931 & 0.905293 & 0.908274 & 0.909249 & 0.945207 & 0.619025 & 0.512318 \\
$:$ & $:$ & & $:$ & & & $\vdots$ & $:$ \\
5.9 & 0.00273944 & 0.00146987 & 0.00838373 & 0.00865573 & 46.3443 & 67.3243 & 68.3511 \\
5.91 & 0.00271219 & 0.00145371 & 0.00834439 & 0.00861477 & 46.4009 & 67.4969 & 68.517 \\
5.92 & 0.0026852 & 0.00143773 & 0.00830535 & 0.00857412 & 46.4574 & 67.669 & 68.6825 \\
5.93 & 0.00265848 & 0.00142192 & 0.00826661 & 0.00853378 & 46.5139 & 67.8407 & 68.8476 \\
5.94 & 0.00263203 & 0.00140629 & 0.00822816 & 0.00849375 & 46.5703 & 68.0119 & 69.0122 \\
5.95 & 0.00260584 & 0.00139082 & 0.00819001 & 0.00845402 & 46.6266 & 68.1827 & 69.1763 \\
5.96 & 0.00257991 & 0.00137553 & 0.00815215 & 0.00841459 & 46.6829 & 68.353 & 69.34 \\
5.97 & 0.00255424 & 0.00136041 & 0.00811458 & 0.00837547 & 46.7392 & 68.5228 & 69.5033 \\
5.98 & 0.00252883 & 0.00134545 & 0.00807729 & 0.00833663 & 46.7953 & 68.6921 & 69.6661 \\
5.99 & 0.00250366 & 0.00133066 & 0.00804028 & 0.00829809 & 46.8515 & 68.861 & 69.8284 \\
6. & 0.00247875 & 0.00131603 & 0.00800355 & 0.00825984 & 46.9075 & 69.0294 & 69.9903 \\
\hline
\end{tabular}

the general solution of the system 4.4 is

$$
\left(\begin{array}{l}
x(t) \\
y(t)
\end{array}\right)=c_{1} v_{1} E_{\alpha}\left(t^{\alpha}\right)+c_{2} v_{2} E_{\alpha}\left(-2 t^{\alpha}\right),
$$

where $c_{1}$ and $c_{2}$ are arbitrary constants. In particular, the initial value problem of system for $\left(\begin{array}{l}x(0) \\ y(0)\end{array}\right)=\left(\begin{array}{l}1.2 \\ 4.2\end{array}\right)$ has the unique solution

$$
\left(\begin{array}{l}
x(t) \\
y(t)
\end{array}\right)=\frac{1}{5}\left(\begin{array}{l}
1 \\
1
\end{array}\right) E_{\alpha}\left(t^{\alpha}\right)+\left(\begin{array}{l}
1 \\
4
\end{array}\right) E_{\alpha}\left(-2 t^{\alpha}\right) .
$$

Figure 2a shows the plots of $x(t)$ versus the time $t$ of the system 4.4 of the exact integration curve (black/solid line) and the approximation time-series for FMDTM (red/dotted line), FAB (green/dashed line), and FABM (blue/dot-dashed line). The value of the fractional order is taken $\alpha=0.98$ as in the previous example. In all numerical simulations, we take the integration step-size $h=0.01$, and the integration time-span is $t \in[0,6]$. Contrary to the previous example, the curves are now increasing towards infinity as $t \rightarrow \infty$, suggesting that the system is unstable. The deviation of the approximation curves from the exact curve is obvious at high values of $t$, which is also seen from the depicted relative difference parameter $R_{x}(t)$ in Fig. 2b. The monotonical increase of the relative difference parameter at large values of $t$ is also observed in the current case, and as before, we have the same non-monotonic behavior at a small time-window in the beginning. The numerical methods gradually diverge from the exact solution as the time rises, and in this case 

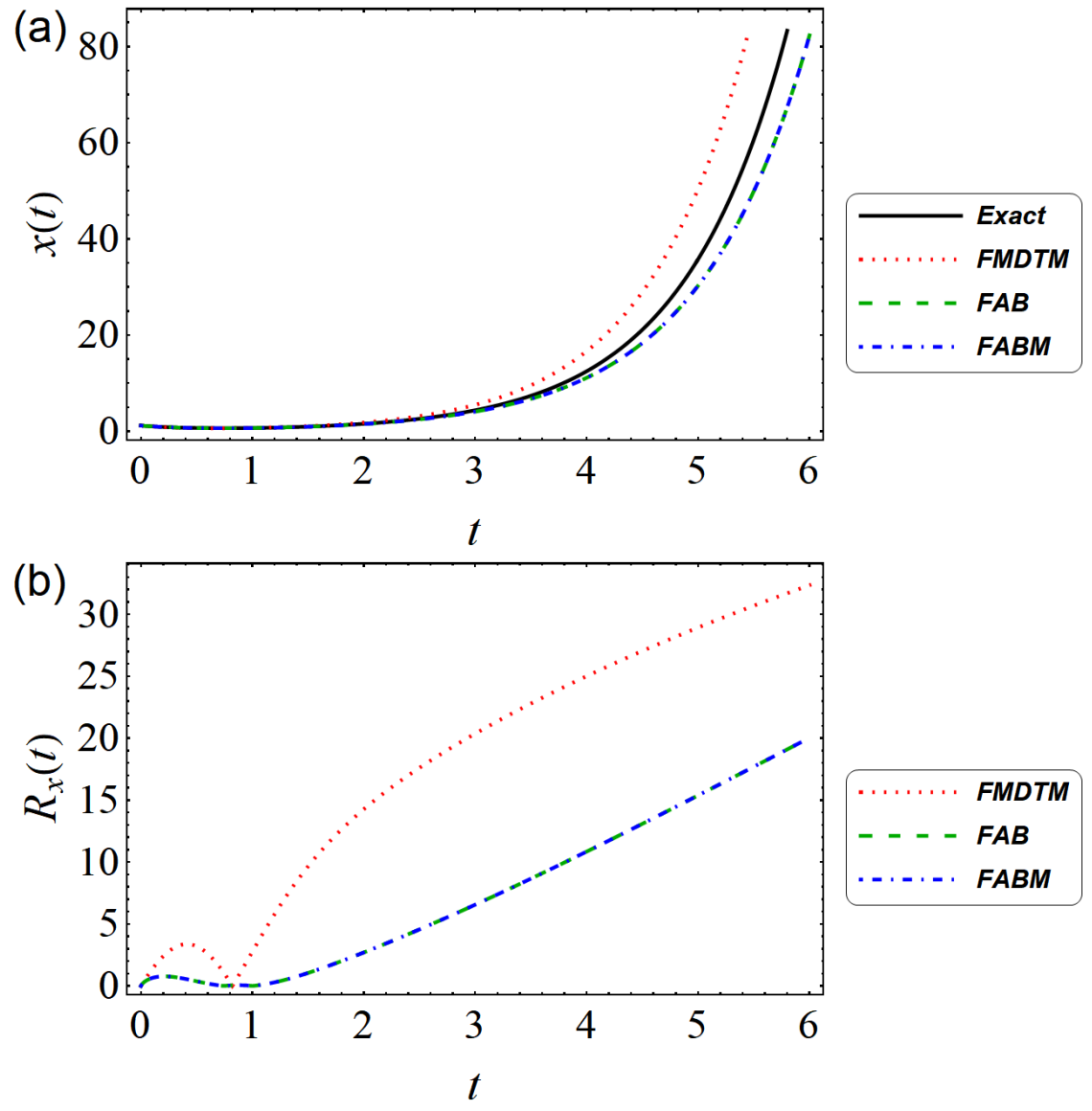

Figure 2. (a). Time-series $x(t)$ versus $t$ of the exact integration curve of Eq. (4.4) (black/solid line) and the approximation curves for FMDTM (dotted/red line), FAB (dashed/green line), and FABM (dot-dashed/blue line); (b). Relative difference $R_{x}(t)$ corresponding to FMDTM (dotted/red line), FAB (dashed/green line), and FABM (dotdashed/blue line). The fractional-order parameter is $\alpha=0.98$. Initial conditions $x(0)=1.2$ and $y(0)=4.2$, integration step size $h=0.01$ and $t \in[0,6]$.

FAB and FABM are slightly better approximations than FMTDM at large time values. The tabluar values of the exact time-series $x_{\text {exact }}$ and the apprroximation time series $x_{F M D T M}, x_{F A B}$ and $x_{F A B M}$, along with the corresponding relative difference parameters $R_{F M D T M}, R_{F A B}$ and $R_{F A B M}$ are provided in Table 2.

The corresponding plots for the second variable $y(t)$ of the system (4.4) are provided in Figs. 3a and 3b, and the tabular values are given in Table 3. The 
TABLE 2. Partial data values of the time-series $x(t)$ for increasing $t$ of the exact solution of system Eq. 4.4 and the numerical approximation values for FMDTM, FAB, and FABM, as well as the corresponding values for the relative difference $R_{x}(t)$ for each numerical method.

\begin{tabular}{c|c|c|c|c|c|c|c}
$t$ & $x_{\text {exact }}$ & $x_{F M D T M}$ & $x_{F A B}$ & $x_{F A B M}$ & $R_{F M D T M}$ & $R_{F A B}$ & $R_{F A B M}$ \\
\hline 0 & 1.2 & 1.2 & 1.2 & 1.2 & 0 & 0 & 0 \\
0.01 & 1.18207 & 1.18036 & 1.18036 & 1.18036 & 0.144451 & 0.144514 & 0.144514 \\
0.02 & 1.16456 & 1.16122 & 1.16175 & 1.16175 & 0.286765 & 0.241222 & 0.241222 \\
0.03 & 1.14747 & 1.14257 & 1.1438 & 1.1438 & 0.426866 & 0.319623 & 0.319623 \\
0.04 & 1.1308 & 1.12441 & 1.12643 & 1.12643 & 0.564677 & 0.385948 & 0.385948 \\
0.05 & 1.11452 & 1.10672 & 1.10958 & 1.10958 & 0.700121 & 0.443174 & 0.443174 \\
0.06 & 1.09865 & 1.08949 & 1.09323 & 1.09323 & 0.83312 & 0.493073 & 0.493073 \\
0.07 & 1.08316 & 1.07272 & 1.07734 & 1.07734 & 0.963596 & 0.536822 & 0.536822 \\
0.08 & 1.06805 & 1.05639 & 1.06191 & 1.06191 & 1.09147 & 0.575268 & 0.575268 \\
0.09 & 1.05332 & 1.0405 & 1.0469 & 1.0469 & 1.21666 & 0.609052 & 0.609052 \\
$:$ & & & & & & & $\vdots$ \\
5.9 & 92.612 & 136.264 & 74.4903 & 74.4903 & 32.0351 & 19.5673 & 19.5673 \\
5.91 & 93.5971 & 137.78 & 75.2389 & 75.2389 & 32.0675 & 19.6141 & 19.6141 \\
5.92 & 94.5927 & 139.312 & 75.995 & 75.995 & 32.0999 & 19.6608 & 19.6608 \\
5.93 & 95.5989 & 140.861 & 76.7587 & 76.7587 & 32.1322 & 19.7075 & 19.7075 \\
5.94 & 96.6158 & 142.427 & 77.5301 & 77.5301 & 32.1646 & 19.7542 & 19.7542 \\
5.95 & 97.6436 & 144.01 & 78.3093 & 78.3093 & 32.1968 & 19.8009 & 19.8009 \\
5.96 & 98.6824 & 145.612 & 79.0963 & 79.0963 & 32.229 & 19.8476 & 19.8476 \\
5.97 & 99.7323 & 147.231 & 79.8912 & 79.8912 & 32.2612 & 19.8944 & 19.8944 \\
5.98 & 100.793 & 148.868 & 80.6941 & 80.6941 & 32.2934 & 19.9411 & 19.9411 \\
5.99 & 101.866 & 150.523 & 81.505 & 81.505 & 32.3255 & 19.9878 & 19.9878 \\
6. & 102.95 & 152.197 & 82.3241 & 82.3241 & 32.3576 & 20.0346 & 20.0346 \\
\hline & & & & & & &
\end{tabular}

conclusions are coincident with the ones we made for the variable $x(t)$ of the system.

\section{Conclusions}

Numerical solutions of the equations governing fractional-order dynamical systems are often the only approach to study the dynamical behavior of these systems at particular parameter values, since in general the exact solutions of differential equations of fractional-order cannot be sought in practice. Therefore, it is of a great significance to choose the right numerical approximation method to describe the correct behavior of the system under investigation [11, 12, 13.

In this paper, we apply three distinct numerical methods (FAB, FABM, and FMDTM) to numerically approximate two different linear systems of fractional differential equations in which fractional derivatives are taken in the sense of Caputo. The linearity of the systems allowed us to compare the numerical results for the three methods with the exact solution for each linear system. To characterise the successfulness of each numerical method, we use the relative difference parameter between the exact solution of each system at a given time point and the approximate solution at the same instance. From the resulting diagrams and tabular values we conclude that, aside from occasional non-monotoncity for small time values, all three numerical methods gradually diverge from the exact solution with increasing integration time. The superiority of each numerical method over the other methods depends on the particular system under investigation: in the 

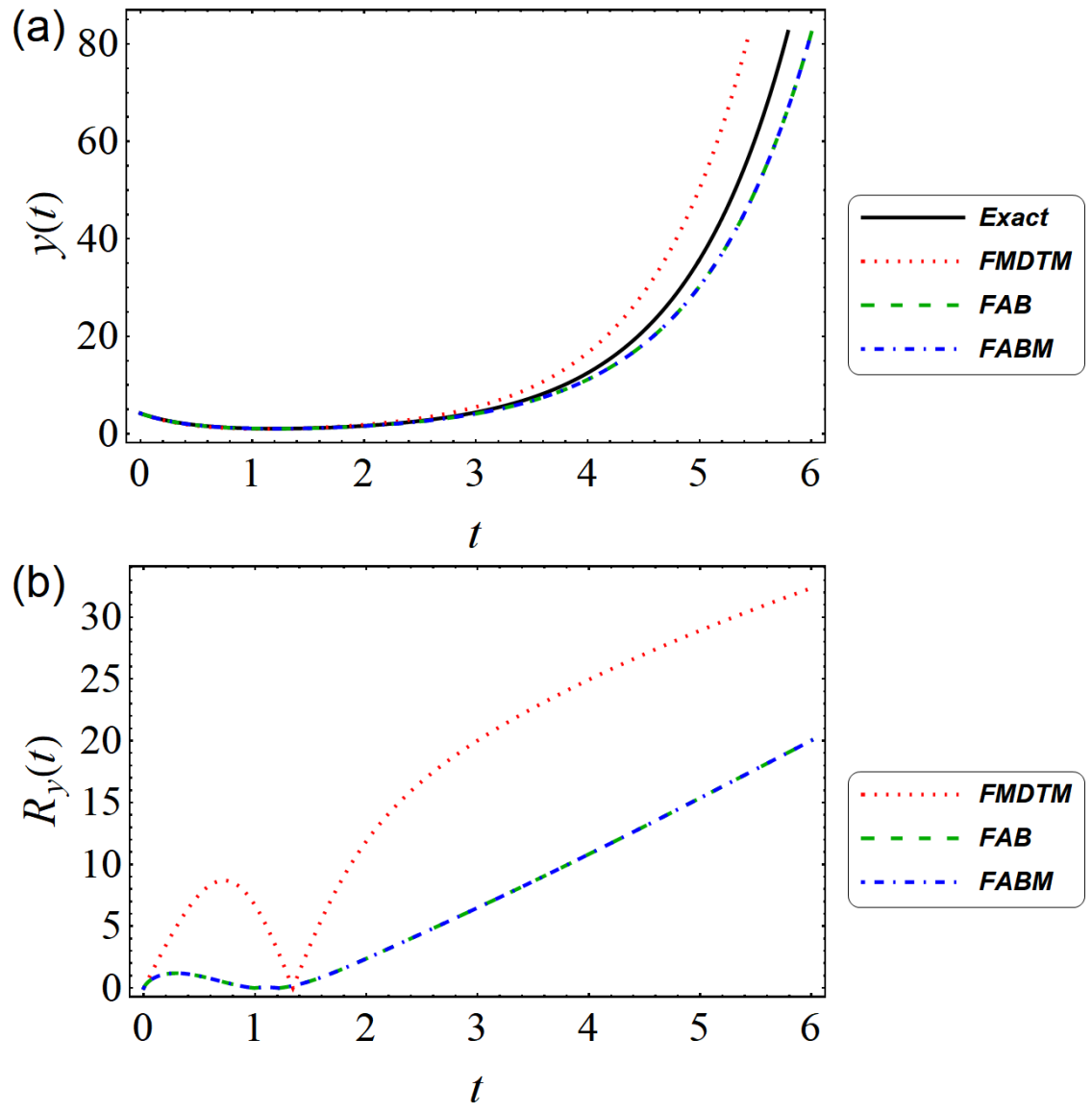

Figure 3. (a). Plots of $y(t)$ versus $t$ of the exact integration curve of Eq. 4.4 (black/solid line) and the approximation curves for FMDTM (dotted/red line), FAB (dashed/green line), and FABM (dotdashed/blue line); (b). Relative difference $R_{y}(t)$ corresponding to FMDTM (dotted/red line), FAB (dashed/green line), and FABM (dotdashed/blue line). The fractional-order parameter is $\alpha=0.98$. Initial conditions $x(0)=1.2$ and $y(0)=4.2$, integration step size $h=0.01$ and $t \in[0,6]$.

first case, FMTDM was shown to be a better approximation than FAB and FABM, and in the second case the situation has been reversed.

More detailed analysis of the three aforementioned numerical methods for additional fractional-order systems that can be solved analytically, and for different integration and system parameters, is an ongoing work in progress. 
TABLE 3. Partial data values of the time-series $y(t)$ for increasing $t$ of the exact solution of system 4.4 and the numerical approximation values for FMDTM, FAB, and FABM, as well as the corresponding values for the relative difference $R_{y}(t)$ for each numerical method.

\begin{tabular}{c|c|c|c|c|c|c|c}
$t$ & $y_{\text {exact }}$ & $y_{F M D T M}$ & $y_{F A B}$ & $y_{F A B M}$ & $R_{F M D T M}$ & $R_{F A B}$ & $R_{F A B M}$ \\
\hline 0 & 4.2 & 4.2 & 4.2 & 4.2 & 0 & 0 & 0 \\
0.01 & 4.12218 & 4.11476 & 4.11476 & 4.11476 & 0.180004 & 0.180063 & 0.180063 \\
0.02 & 4.04601 & 4.03146 & 4.03377 & 4.03377 & 0.359603 & 0.30257 & 0.30257 \\
0.03 & 3.97145 & 3.95006 & 3.95542 & 3.95542 & 0.538766 & 0.403653 & 0.403653 \\
0.04 & 3.89848 & 3.87051 & 3.87934 & 3.87934 & 0.717459 & 0.490837 & 0.490837 \\
0.05 & 3.82705 & 3.79277 & 3.80532 & 3.80532 & 0.895647 & 0.567682 & 0.567682 \\
0.06 & 3.75714 & 3.71681 & 3.73323 & 3.73323 & 1.07329 & 0.636283 & 0.636283 \\
0.07 & 3.68872 & 3.64259 & 3.66297 & 3.66297 & 1.25037 & 0.698022 & 0.698022 \\
0.08 & 3.62175 & 3.57007 & 3.59445 & 3.59445 & 1.42682 & 0.75388 & 0.75388 \\
0.09 & 3.55621 & 3.49922 & 3.5276 & 3.5276 & 1.60262 & 0.804593 & 0.804593 \\
$:$ & $:$ & & $\vdots$ & $:$ & & & $\vdots$ \\
5.9 & 92.6183 & 136.264 & 74.4969 & 74.4969 & 32.0304 & 19.5657 & 19.5657 \\
5.91 & 93.6034 & 137.78 & 75.2455 & 75.2455 & 32.0629 & 19.6124 & 19.6124 \\
5.92 & 94.599 & 139.312 & 76.0016 & 76.0016 & 32.0954 & 19.6591 & 19.6591 \\
5.93 & 95.6052 & 140.861 & 76.7653 & 76.7653 & 32.1278 & 19.7059 & 19.7059 \\
5.94 & 96.6221 & 142.427 & 77.5367 & 77.5367 & 32.1602 & 19.7526 & 19.7526 \\
5.95 & 97.6499 & 144.01 & 78.3159 & 78.3159 & 32.1925 & 19.7994 & 19.7994 \\
5.96 & 98.6887 & 145.612 & 79.1028 & 79.1028 & 32.2248 & 19.8461 & 19.8461 \\
5.97 & 99.7385 & 147.231 & 79.8977 & 79.8977 & 32.257 & 19.8928 & 19.8928 \\
5.98 & 100.8 & 148.868 & 80.7006 & 80.7006 & 32.2892 & 19.9396 & 19.9396 \\
5.99 & 101.872 & 150.523 & 81.5115 & 81.5115 & 32.3214 & 19.9863 & 19.9863 \\
6. & 102.956 & 152.197 & 82.3306 & 82.3306 & 32.3535 & 20.0331 & 20.0331 \\
\hline
\end{tabular}

\section{REFERENCES}

[1] S. K. Miller, B. Ross, An introduction to the fractional calculus and fractional differential equations, (1993), John Wylei and Sons, Inc., Canada.

[2] D. Baleanu, K. Deithelm, E. Scalas, J. J. Trujillo, Fractional Calculus: models and numerical methods, (2017), World Scientific, Singapore.

[3] L. Changpin, Z. Fanhai, Numerical Methods for Fracional Calculus, (2015), CRC Press Taylor and Francis Group, New York.

[4] K. Diethelm, N. J. Ford and A. D. Freed, Detailed error analysis for a fractional Adams method, Numerical Algorithms, 36, (2004), 31-52.

[5] K. Diethelm, N. J. Ford and A. D. Freed, A Predictor-Corrector Approach for the Numerical Solution of Fractional Differential Equations, Nonlinear Dynamics, 29, (2001), 3-22.

[6] C. Bervillier, Status of the diffrential transformation method, Applied Mathematics and Computation, 20, (2012), 10158-10170.

[7] S. V. Ertürk, Sh. Momani, Solving systems of fractional differential equations using differential transform method, Journal of Computational and Applied Mathematics, 215, (2008), $142-151$.

[8] R. Garrappa, On some explicit Adams multistep methods for fractional differential equations, Journal of Computational and Applied Mathematics, 229, (2009), 392-399.

[9] Z. M. Odibat and S. Momani, An algorithm for the numerical solution of differential equations of fractional order, Journal of Applied Mathematics and Informatics, 26, (2008), 15-27.

[10] Z. M. Odibat, Analytic study on linear systems of fractional differential equations, Computers and Mathematics with Applications, 59, (2010), 1171-1183.

[11] G. Markoski, Bifurcation Analysis of fractional-order Chaotic Rossler System, Matematichki Bilten, 42, (2018), 28-37.

[12] Y. Seferi, G. Markoski and A. Gjurchinovski, Comparison of different numerical methods for fractional differential equations, Matematichki Bilten, 42, (2018), 61-74. 
[13] Y. Seferi, G. Markoski and A. Gjurchinovski, Comparison of two numerical methods for fractional-order Rossler system, Matematichki Bilten, 44, (2020), 53-60.

1 University of Tetova, North Macedonia

Email address: ylldrita.seferi@unite.edu.mk

2 Institute of Mathematics,

Faculty of Natural Sciences and Mathematics,

Ss. Cyril and Methodius University, Skopje, North Macedonia

Email address: gorgim@pmf.ukim.mk

3 Institute OF PHYSiCs,

Faculty of Natural Sciences and Mathematics,

Ss. Cyril and Methodius University, Skopje, North Macedonia

Email address: agjurcin@yahoo.com

Received: 8.2 .2021

Revised: 12.3 .2021

Accepted: 15.3.2021 